\title{
One-dimensional electron lattice system with a long-range interelectron repulsion on a disordered host lattice
}

\author{
A.A. Slutskin and H.A. Kovtun \\ B. Verkin Institute for Low Temperature Physics and Engineering of the National Academy \\ of Sciences of Ukraine, 47 Lenin Ave., Kharkov 61103, Ukraine \\ E-mail: slutskin@theor.kharkov.ua \\ kovtun@theor.kharkov.ua
}

Received November 29, 2004

\begin{abstract}
We study what happens to generalized Wigner crystal, GWC (a regular structure formed by narrow-band electrons on a one-dimensional periodic host lattice), when there is a host lattice random distortion that does not break the host-lattice long-range order. We show that an arbitrarily weak distortion of the kind gives rise to soliton-like GWC defects (discrete solitons, DS) in the ground state, and thereby converts the ordered GWC into a new disordered macroscopic state lattice Wigner glass (LWG). The ground-state DS concentration is found to be proportional to $\lambda^{4}$ ( $\lambda$ is the typical host-lattice strain). We show that the low-temperature LWG thermodynamics and kinetics are fully described in DS terms. A new phenomenon of a super-slow logarithmic relaxation in the LWG is revealed. Its time turns out to be tens orders of magnitude greater than the microscopic ones. Analytical dependences of LWG thermodynamic quantities on temperature and $\lambda$ are obtained for an arbitrary relationship between the relevant Coulomb energies and the electron bandwidth.
\end{abstract}

PACS: 73.61.Jc, 75.10.Nr

\section{Introduction}

In the last two decades layered and low-dimensional conductors have been a focus of attention. In such systems the charge carriers are commonly well-separated from the dopants, so that a mutual Coulomb repulsion of the charge carriers turns out to be an essential factor. Among these conductors of especial interest are lattice (narrow-band) systems wherein hopping (tunneling) of electrons/holes between host-lattice sites is suppressed by their mutual repulsion, and as a consequence of this, the whole charge carrier ensemble is self-localized. The criterion for such a Coulomb self-localization (CSL) is the inequality

$$
t<u_{c} \sim\left(a_{0} / r_{e e}\right)^{2} \varepsilon_{e e},
$$

where $t$ is the bandwidth, $u_{c}$ is the typical change of the Coulomb energy of a charge carrier as it hops between neighboring host-lattice sites, $a_{0}$ is the hostlattice spacing, $r_{e e}$ is the mean separation between charge carriers, and $\varepsilon_{e e}$ is the mean energy of the
Coulomb repulsion per charge carrier. There exists a wide class of CSL conductors. Different semiconductor heterostructures [1,2], and organic quasi-onedimensional conductors [3] fall into this class. The latest achievements of the nanotechnology permit creation of CSL conductors of a new type: arrays of quantum dots exchanging electrons/holes (see, for example, [4] and references there), electrons on helium surface [5], and nets and chains of metal nano-grains linked by organic molecule wires as (weak) tunnel junctions [6]. There are also good reasons to suggest that the CSL criterion is fulfilled in layered metaloxides of a type of high-temperature superconductors, provided the dopant concentration is not too low.

Impetus to a theoretical research of CSL conductors was given by Hubbard [3], who considered the ground state of an electron ensemble on a one-dimensional (1D) periodic host lattice in the limit $t / u_{c} \rightarrow 0$. Hubbard suggested that in the thermodynamic limit (the numbers of electrons $N$ and host-lattice sites $N_{s}$ tend to $\infty$ ) the ground-state electron structure at a given chemical potential $\mu$ is a periodic «generalized Wigner crystal» (GWC) with a rational 
electron density (filling factor) $n_{e}=P / Q(P, Q$ are arbitrary coprime integers), with spacing $Q a_{0}$ and $P$ electrons per unit cell, which does not depend on the pair potential of the interelectron repulsion $v(x)$ ( $x$ is the interelectron distance), provided $v(x)$ meets some simple physically reasonable conditions (Sec. 2). Hubbard also postulated a universal algorithm to find GWC's. This hypothesis was justified in works $[7,8]$, wherein Hubbard's algorithm was put into a compact form. In recent years Hubbard's results were augmented to the low-temperature thermodynamics of $1 \mathrm{D}$ GWC [9], and the ground state of two-dimensional (2D) CSL conductors on an arbitrary ideal host lattice [10]. The regular 2D CSL conductor was found to be characterized by an effective lowering of dimension, which lies in the fact that even in the case of an isotropic pair potential of electron-electron repulsion the structure elements forming the 2D ground-state structure are electron stripes arranged by Hubbard's algorithm [10].

The above studies, having revealed a number of unusual features of GWC and its 2D modification, have prompted consideration of the influence of host-lattice disorder on low-temperature properties of CSL conductors. This is of importance not only because there are a number of disordered CSL conductors (e.g., MOSFETs, some nanostructures), but also in respect to the known fact (Larkin [11], Imri and Ma [12]) that an arbitrarily weak static random peturbation imposed on a continuous Wigner crystal (i.e., a crystal formed by electrons that are free to move) breaks (for dimension $d \leq 3$ ) the ground-state crystalline long-range order, producing slowly varying random distortions that become increasingly smooth as the perturbation goes to zero. Such a structure was named «Wigner glass». Unlike the continuous case, the electron displacements in CSL conductors cannot be less than the host-lattice spacing $a_{0}$, so that the Larkin-Imri-Ma argument is inapplicable to clarify whether lattice electron structures are unstable with respect to weak random perturbations, and what is the mechanism of the possible instability. As far as we know, there is no answer to this question at present. We are going to fill this gap, considering the low-temperature properties of disordered $1 \mathrm{D}$ and 2D CSL conductors in a series of publications. This paper is the first of the series. Here we study the ground state, elementary excitations, their lifetimes, and the low-temperature thermodynamics of a 1D CSL conductor (the temperature $T \ll u_{c}$ ) in the limit of strong CSL $\left(t<<u_{c}\right)$ with the assumption that random host-lattice distortions do not break long-range order of the arrangement of host-lattice sites. For definiteness, the charge carriers are further considered to be electrons.
Specifically, we consider the host lattice to be a chain of sites $i=1, \ldots, N_{s}$ with coordinates

$$
X_{i}=i+\lambda \xi_{i}
$$

where $\lambda \xi_{i}$ is a random deviation of the $i$-th site from its ideal position $X_{i}^{0}=i\left(a_{0}=1\right) ; \xi_{i}$ as a function of $i$ takes random values on the interval $[-1,1]$; the disorder parameter $\lambda$ is assumed to be $<1 / 2$ to provide the periodic correlation in the host lattice. At first glance such a disorder cannot break the GWC long-range order. However, this seemingly evident statement turns out to be untrue: we show here that for any finite $\lambda<<1$ the ground-state electron configuration contains soliton-like GWC defects, discrete solitons, each changing the length of the system (at a given $N$ ) by +1 (the defect of rarefaction) or -1 (the defect of compression). Being randomly arranged, they do break the GWC long-range order. This is a novel phenomenon rooted in the discretness of the system. The key point is that the discrete solitons differ from common point defects in that a transfer of a discrete soliton over $\mathcal{L}$ unit cells of a GWC leaves behind a «trace» - a cluster of electrons shifted by one host-lattice site from their ground-state positions, the number of the electrons being $\mathcal{L}$. Owing to the hostlattice disorder the Coulomb energy of the trace is a sum of $\mathcal{L}$ random alternating-sign quantities $\propto \lambda^{2}$, which grows in modulus as $\sqrt{\mathcal{L}}$, attaining values $\sim u_{c}$ for sufficiently big $\mathcal{L}$. It is this fact that accounts for discrete solitons coming into existence in the ground state. Since the random 1D lattice electron structure just described is bound to be converted into GWC as $\lambda \rightarrow 0$, it can be conceived of, by anology with the (continual) Wigner glass, as a «lattice Wigner glass» (LWG). As will be seen from the following, the «giant» Coulomb energy fluctuations caused by discrete-soliton transfer underlie the distinctive features of both LWG thermodynamics and kinetics. They have nothing in common with the energy fluctuations in the continuous Wigner glass that result from the above-mentioned smooth random distortions. Therefore, the macroscopic behavior of LWG is expected to differ essentially from that of Wigner glass. This is dramatically manifested in relaxation of high-energy LWG excitations: our analysis shows that their lifetimes are anomalously great, the concentration of the excitations decreasing with time in a logarithmic fashion. Broadly speaking, the relaxation is by annihilation of GWC defects of compression and rarefaction. This requires the defects to overcome (by tunneling or in a thermal way) a giant fluctuation energy barrier of many-electron origin. The phenomenon is somewhat reminiscent of the mechanism of the nonergodic behavior of spin glass [13,14]. 
The layout of the material in our paper is as follows. In the next Section the Hamiltonian of the system under consideration is described. In Sec. 3 we show that for an arbitrary small $\lambda$ the GWC long-range order is unstable with respect to formation of GWC defects at zero temperature, and find the ground-state dependence of the GWC defects concentration on $\lambda$ and $\mu$ in the limit $t=0$. The LWG low-temperature thermodynamics $\left(T<<u_{c}\right)$ is considered in Sec. 4, where the dependence of thermodynamic quantities on $T$ is found in terms of low-lying LWG excitations. The anomalously slow relaxation in LWG is discussed in Sec. 5. In the last Section we discuss experimental possibilities for studing LWG.

\section{Hamiltonian}

At a given $\mu$ the general Hamiltonian $\mathcal{H}$ of an electron system on an $1 \mathrm{D}$ host lattice is of the form

$$
\begin{gathered}
\mathcal{H}=t \sum_{i}\left(c_{i}^{+} c_{i-1}+\text { h.c. }\right)+ \\
+\frac{1}{2} \sum_{i \neq i^{\prime}} v\left(X_{i}-X_{i^{\prime}}\right) n_{i} n_{i^{\prime}}-\mu \sum_{i} n_{i} .
\end{gathered}
$$

Here the index $i$ enumerates host-lattice sites, $c_{i}^{+}$and $c_{i}$ are operators of creation and annihilation of an electron at the $i$-th site, respectively; $n_{i}$ is the operator of the electron density at the $i$-th site, which takes only values 0 and 1 , since $n_{e}$ is assumed to be not too close to $1 / 2$; for this reason the electron spin indexes are dropped; summation is over all host lattice sites; the pair potential of an electron-electron repulsion $v(x)=v(-x)>0$. The dependence of the site coordinate $X_{i}$ on $i$ is given by Eq. (1).

To find the ground-state structure in the $t<<u_{c}$ limit under consideration, one can put $t=0$, the Hamiltonian Eq. (2) reducing to

$$
\mathcal{H}=\mathcal{H}\{i(m)\}=\frac{1}{2} \sum_{m \neq m^{\prime}} v\left(x_{m}-x_{m^{\prime}}\right)-\mu N,
$$

where the index $m=1, \ldots, N$ enumerates the electrons, $x_{m}=X_{i(m)}$ is the coordinate of the $m$-th electron, and $i(m)$ is the number of the site at which the $m$-th electron is localized. The reduced Hamiltonian Eq. (3) is a functional of electron configurations $\{i(m)\}=i(1), \ldots, i(N)$. It differs from that considered by Hubbard [3] only in that $X_{i}$ contains the random addition $\lambda \xi_{i}$. To proceed with our studies it is of benefit to formulate briefly, following $[3,7,8]$, the main properties of the ground state at $\lambda=0$.

Below we consider the pair potential $v(x)>0$ to be an everywhere convex function of $x$ that goes to zero with an increase of $x$ faster than $x^{-1}$; its dependence on $x$ is otherwise arbitrary. In such a case the ground-state space structure of the 1D electron gas on the ideal host lattice $X_{i}^{0}=i$ is given by the above-mentioned universal Hubbard's algorithm that is expressed by the simple formula

$$
i(m)=i_{H}\left(m, n_{e}\right) \equiv\left[m / n_{e}+\varphi\right],
$$

where $[\ldots]$ is the integer part of a number, and $\varphi$ is an arbitrary phase that reflects the arbitrariness of the choice of the first electron site coordinate $i(1)$. As was mentioned in the Introduction, only rational $n_{e}=P / Q$ survive at zero temperature and a given $\mu$, the function $i_{H}(m, P / Q)$ determining the GWC. The ground-state dependence of $n_{e}$ on $\mu$ is a devil's staircase: for each pair $P, Q$ there is an interval $\mu_{-}(P, Q) \leq \mu \leq \mu_{+}(P, Q)$ within the limits of which $n_{e}(\mu)$ remains equal to $P / Q$. The left and right endpoints of the devil's-staircase interval is a decrease and an increase in the Coulomb energy of the system as $N$ changes by -1 and +1 , respectively. The length of the interval $\Delta \mu=\mu_{+}-\mu_{-}$depends only on $Q$ :

$$
\begin{gathered}
\Delta \mu=Q u_{Q}, \\
u_{Q}=\sum_{k=1}^{\infty} k[v(Q k+1)-2 v(Q k)+v(Q k-1)] .
\end{gathered}
$$

As is seen from this expression, $u_{Q} \sim u_{c}$, and hence, $\Delta \mu$ vanishes rapidly together with $u_{c}$ as $Q$ tends to $\infty$.

\section{The ground state of LWG}

\subsection{GWC instability}

In this Section we deal with the Hamiltonian of Eq. (3). First we consider devil's-staircase intervals with $n_{e}=1 / Q$. Generalization to an arbitrary $n_{e}=$ $=P / Q$ is performed without difficulty (Sec. 5). In the case of $P=1$ the Hubbard's algorithm Eq. (4) gives the simple periodic dependence $i_{H}(m, 1 / Q)=i_{m}^{v} \equiv$ $\equiv Q m+v$ with the $v=0, \ldots, Q-1$. (We will call this the « $v$-configuration».) If the ground-state electron arrangement $i_{g}(m)$ was the same as in the GWC (i.e., $\left.i_{g}(m)=i_{m}^{v}\right)$ despite the random small displacements $\lambda \xi_{i}(\lambda<<1)$, a weak disorder of the form Eq. (1) would affect only the energy of the $v$-configuration $E_{\mathrm{GWC}}$. The latter does not depend on $v$ to an accuracy of fluctuation corrections $\propto \sqrt{N}$ :

$$
\begin{gathered}
E_{\mathrm{GWC}}=N \epsilon+\mathcal{O}(\sqrt{N}), \\
\epsilon=\epsilon_{0}+\epsilon_{\lambda}, \quad \epsilon_{\lambda}=\lambda^{2}\left\langle\xi^{2}\right\rangle \sum_{k=1}^{\infty} v^{\prime \prime}(k Q) .
\end{gathered}
$$


Here $\epsilon$ is the mean energy per electron, $\epsilon_{0}$ is that for the ideal host lattice, $\epsilon_{\lambda}$ is the addition to $\epsilon_{0}$ produced by a weak host-lattice disorder, $\langle\ldots\rangle$ is the average on the $\xi$ distribution, and $v^{\prime \prime}(x)=d^{2} v / d x^{2}$.

Let us show that the periodic (in site coordinates) $v$-configuration is unstable under conditions of a weak disorder. To reveal this, let us find out what happens to the energy Eq. (3) if some v-configuration cluster formed by electrons with numbers $m_{1}, \ldots, m_{2}$ is shifted as a whole by $\pm 1: i_{m}^{v} \rightarrow i_{m}^{v \pm 1}, m_{1} \leq m \leq m_{2}$.

The energy of the cluster $E\left(m_{1}, m_{2}, v\right)$ is the sum

$$
E\left(m_{1}, m_{2}, v\right)=\epsilon_{b}+E_{i}\left(m_{1}, m_{2}, v\right)
$$

whose first term is the cluster boundary energy, i.e., the energy of the interaction of the cluster with its surroundings. The second term is the internal cluster energy:

$$
E_{i}\left(m_{1}, m_{2}, v\right)=\left(m_{2}-m_{1}\right) \epsilon+\tilde{E}\left(m_{1}, m_{2}, v\right) ;
$$

$\tilde{E}\left(m_{1}, m_{2}, v\right)$ is its fluctuating part. Expanding energy Eq. (3) in powers of $\lambda$, we obtain

$$
\begin{gathered}
\tilde{E}\left(m_{1}, m_{2} ; v\right)=\sum_{m=m_{1}}^{m_{2}}\left(\epsilon_{m}^{v}-\epsilon\right), \\
\epsilon_{m}^{v}=\frac{\lambda^{2}}{2} \sum_{m^{\prime}=-\infty}^{\infty} v^{\prime \prime}\left(Q\left(m-m^{\prime}\right)\right)\left[\xi\left(i_{m}^{v}\right)-\xi\left(i_{m^{\prime}}^{v}\right)\right]^{2}+ \\
+\mathcal{O}\left(\lambda^{3}\right) .
\end{gathered}
$$

The shift of the cluster, on the one hand, creates two defects at the ends of the cluster, which are pairs of electrons separated by distances $Q+1$ (rarefaction defect, «-dimer») and $Q-1$ (compression defect, «+dimer»). Using expression (5), one can obtain that in the case of \pm dimers well-separated dimer formation increases the cluster boundary energy by

$$
\delta \epsilon_{b}=\Delta \mu / Q+\tilde{\epsilon}_{-}(m, v)+\tilde{\epsilon}_{+}(m, v),
$$

where $\Delta \mu / Q$ is the total energy of dimer formation at $\lambda=0 ; \tilde{\epsilon}_{ \pm}(m, v)$ is a small $\left(\sim \lambda u_{c}\right)$ random addition produced by the host-lattice disorder. On the other hand, the shift changes $\tilde{E}\left(m_{1}, m_{2} ; v\right)$ by

$$
\Delta_{v, v^{\prime}}=\Delta_{v, v^{\prime}}\left(m_{1}, m_{2}\right)=\sum_{m=m_{1}}^{m_{2}}\left(\epsilon_{m}^{v^{\prime}}-\epsilon_{m}^{v}\right), v^{\prime}=v \pm 1 .
$$

This energy change, being a sum of random quantities $\epsilon_{m}^{v^{\prime}}-\epsilon_{m}^{v}$ with the zero average, fluctuates in $m_{1}, m_{2}$, taking both positive and negative values. Its modulus $\left|\Delta_{v, v^{\prime}}\right| \sim \lambda^{2} u_{c}\left(m_{2}-m_{1}\right)^{1 / 2}$ therewith grows as $m_{2}-m_{1}$ increases. Therefore, despite the smallness of $\lambda$ there are many sufficiently long clusters for which
$\Delta_{v, v^{\prime}}+\delta \epsilon_{b}<0$, and hence, their shifts decrease the energy of the system. What it means is that the GWC is unstable relative to dimer formation for an arbitrarily small $\lambda$.

\subsection{The space structure of the $L W G$ ground state}

As follows from the general theory of GWCs $[3,7,8]$ (see also [9]), in the case under consideration $(P=1)$ the \pm dimers are the GWC defects with the least energies of formation at a given pressure. This fact together with the above reasoning suggests that for any finite $\lambda<<1$ the ground-state configuration $i_{g}(m)$ consists of long periodic electron clusters, segments of v-configurations, alternating with \pm dimers. One should thus seek $i_{g}(m)$ among functions $i(m)$ of the form

$$
\begin{aligned}
& i(m)=Q m+v^{\alpha}\left(m_{1}^{\alpha} \leq m \leq m_{2}^{\alpha}\right), \\
& m_{1}^{\alpha}=m_{2}^{\alpha-1}+1, v^{\alpha}=v^{\alpha-1}-\sigma_{\alpha},
\end{aligned}
$$

where index $\alpha$ enumerates periodic clusters of the configuration $i(m), \sigma_{\alpha}=-1$ or 1 depending on whether $(\alpha-1)$-th and $\alpha$-th clusters are joined by - or + dimer, respectively. The coordinates $m_{1 g}^{\alpha}, m_{2 g}^{\alpha}$ of the end electrons of ground-state clusters are obviously random, and, hence, there is no long-range interelectron correlation in the ground state. This is just the LWG mentioned in the Introduction, the discrete solitons being \pm dimers. The LWG correlation radius $L_{g} \sim 1 /\left(n_{-}^{g}+n_{+}^{g}\right)$ (in units of $\left.r_{e e}=Q\right)$, where $n_{ \pm}^{g}=\mathcal{N}_{ \pm}^{g} / N$ is the ground-state concentration of \pm dimers, $\mathfrak{N}_{ \pm}^{g}$ is their number in the ground state.

The next step is to find $n_{ \pm}^{g}$. To this end one should consider the general expression for the energy $E$ of the configuration (12). It is appropriate to compare $E$ with $E_{\mathrm{GWC}}$ (Eq. (6)), mapping the configuration on a set of $v$-configurations. In so doing $\alpha$-th cluster is considered as the cluster of $\bar{v}^{\alpha}$-configuration $\left(\bar{v}^{\alpha}=v^{\alpha}-Q\left[v^{\alpha} / Q\right]\right.$ is the reduced phase less than $\left.Q\right)$ with the end electrons $\bar{m}_{1,2}^{\alpha}=m_{1,2}^{\alpha}+\left[v^{\alpha} / Q\right]$. The mapping gives

$$
E=E_{\mathrm{GWC}}+E_{\mathrm{dim}}+\tilde{E},
$$

where $E_{\text {dim }}$ is the total energy of dimer formation, and $\tilde{E}$ is the fluctuating part of the energy.

To the zero approximation in $\lambda$

$$
E_{\text {dim }}=\left(n_{-} u_{-}+n_{+} u_{+}\right) N
$$

( $n_{ \pm}=\mathcal{N}_{ \pm} / N$ is the \pm dimer concentration for a given configuration (12), $\mathcal{X}_{ \pm}$is the number of the \pm dimers), and 


$$
\begin{gathered}
u_{ \pm}(\mu)=\frac{u_{Q}}{2}(1 \mp z)>0 \\
\left(z=2(\mu-\bar{\mu}) / \Delta \mu, \bar{\mu}=\frac{\mu_{-}+\mu_{+}}{2}\right)
\end{gathered}
$$

are the \pm dimer formation energies at a given $\mu$; the variable $z$ takes values on the interval $[-1,1]$ as $\mu$ is varied from $\mu_{-}$to $\mu_{+} ; u_{Q}$ has been defined in Eq. (5). Writing Eqs. (14),(15), we allow for the fact that a small variation $\pm \delta n_{e}$ of $n_{e}$ about $n_{e}=1 / Q$ gives rise to $Q \delta n_{e} \pm$ dimers (this follows immediately from Eq. (4)), i.e.,

$$
N=N_{0}+Q\left(\mathcal{N}_{+}-\mathcal{N}_{-}\right)
$$

$N_{0}=N_{s} / Q$ is the ground-state number of electrons at $\lambda=0$.

The energy $\tilde{E}$, unlike the two previous terms in Eq. (13), depends upon the dimer coordinates $m_{1}^{\alpha}$ essentially:

$$
\tilde{E}=\sum_{\alpha} \tilde{E}\left(\bar{m}_{1}^{\alpha}, \bar{m}_{2}^{\alpha} ; \bar{v}^{\alpha}\right)
$$

As follows from Eq. (9), the quantities $\tilde{E}\left(\bar{m}_{1}^{\alpha}, \bar{m}_{2}^{\alpha} ; \bar{v}^{\alpha}\right)$ are sums of $l_{\alpha}=m_{2}^{\alpha}-m_{1}^{\alpha} \sim\left(n_{-}+n_{+}\right)^{-1 / 2}$ random terms with zero average and moduli $\sim \lambda^{2} u_{c}$. Since $\tilde{E}\left(\bar{m}_{1}^{\alpha}, \bar{m}_{2}^{\alpha} ; \bar{v}^{\alpha}\right)$ fluctuate randomly, it is easy to conclude that in the limit $n_{ \pm}<<1$ the cluster endpoints $m_{1}^{\alpha}, m_{2}^{\alpha}$ can be chosen in such a way that terms of the sum (17) are all negative, and hence, at a given $n_{ \pm}$ the minimum $\tilde{E}_{\min }$ of $\tilde{E}$ with respect to dimer arrangement is bound to be $<0$.

Random sequences $\epsilon_{1}^{v}, \epsilon_{2}^{v}, \ldots, \epsilon_{N}^{v} \quad(v=0, \ldots, Q-1)$, whose terms are involved in expression (9), are all statistically equivalent. Therefore, $\tilde{E}_{\min }$ does not depend on the sequence $\left\{\sigma_{\alpha}\right\}=\sigma_{1}, \sigma_{2}, \ldots$, being a function of the dimer density $n_{-}+n_{+}$and system parameters only. Taking this into account together with the fact that $\left|\tilde{E}\left(\bar{m}_{1}^{\alpha}, \bar{m}_{2}^{\alpha} ; \bar{v}^{\alpha}\right)\right| \sim \lambda^{2} u_{c} l_{\alpha}^{1 / 2}$, one can write $\tilde{E}_{\text {min }}$ as

$$
\tilde{E}_{\text {min }}=-u_{\xi} \lambda^{2}\left(n_{-}+n_{+}\right)^{1 / 2} N
$$

where energy factor $u_{\xi} \sim u_{c}$ is uniquely determined by $Q, v(x)$, and the moments of the $\xi$ distribution (see below).

The ground-state concentrations of \pm dimers, $n_{+}^{g}$, are those which minimize the sum $\tilde{E}_{\text {min }}+E_{\text {dim }}$. Using Eqs. (14), (15), and (18), we find that for any $\lambda \neq 0$

$$
n_{-}^{g}(z)=\left\{\begin{array}{lc}
\frac{u_{\xi}^{2}}{u_{Q}^{2}} \frac{\lambda^{4}}{(1+z)^{2}}, & -1<z<0 \\
0, & 0<z<1
\end{array} ;\right.
$$

$$
n_{+}^{g}(z)=\left\{\begin{array}{lc}
0, & -1<z<0 \\
\frac{u_{\xi}^{2}}{u_{Q}^{2}} \frac{\lambda^{4}}{(1-z)^{2}}, & 0<z<1
\end{array} .\right.
$$

It remains to find the explicit form of $u_{\xi}$. This requires rather sophisticated calculations. Here we only outline them. Since $u_{\xi}$ does not depend on $\left\{\sigma_{\alpha}\right\}$, it is convenient to choose the sequence $\left\{\sigma_{\alpha}\right\}$ with alternating $\sigma_{\alpha}$ signs, considering the minimum of $\tilde{E}+u \mathcal{N}$ instead that of $\tilde{E}$; here $u$ is a given arbitrary positive parameter, and $\mathcal{N}=\mathcal{N}_{-}=\mathcal{N}_{+}$is the variable that should be found as the result of the minimization. This value, $\mathcal{N}=\overline{\mathcal{N}}$, can be calculated in terms of a random walk of the sum $\Delta_{0,1}(1, m)$ (see expression $(11)$ ) as a function of $m$. So doing, one should take into account that the $\tilde{E}+u \mathcal{N}$ minimum is realized if dimers of a given sign are all positioned at the points $m_{\alpha}(\alpha=1,2, \ldots, \mathcal{N})$ such that $\Delta_{0,1}(1, m)-\Delta_{0,1}\left(1, m_{\alpha}\right)$ first attains $u$ value at some points $m=m_{\alpha}^{r}>m_{\alpha}$ (a random walk toward the right) and $m=m_{\alpha}^{l}<m_{\alpha}$ (a random walk toward the left) without taking zero value on intervals $\left[m_{\alpha}, m_{\alpha}^{r}\right]$ and $\left[m_{\alpha}^{l}, m_{\alpha}\right]$. The total number $\overline{\mathcal{N}}$ of points $m_{\alpha}$ is found on the basis of the probability theory methods [15] that are used in consideration of the so-called first passage and renewal problems. The factor $u_{\xi}$ is obtained in view of the fact that the minimum of $\tilde{E}+u \mathcal{N}$ is also $\min _{\mathcal{N}}\left(-u_{\xi} \mathcal{N}^{1 / 2}+u \mathcal{N}\right)$. The net result of the calculation is the following:

$$
u_{\xi}=\left(D / 2 \pi^{2}\right)^{1 / 2} ;
$$

$D \sim u_{c}^{2}$ is the diffusion coefficient for the random walk of $\Delta_{v, v^{\prime}} / \lambda^{2}$, which is given by the formula

$$
\begin{gathered}
D=v_{1}^{2}\left(<\xi^{4}>-<\xi^{2}>^{2}\right)+v_{2}\left(<\xi^{4}>+3<\xi^{2}>^{2}\right) ; \\
v_{1}=\sum_{\substack{k=-\infty \\
k \neq 0}}^{\infty} v^{\prime \prime}(Q k), \quad v_{2}=\sum_{\substack{k=-\infty \\
k \neq 0}}^{\infty}\left(v^{\prime \prime}(Q k)\right)^{2} .
\end{gathered}
$$

Existence of dimers in the ground state not only renders a long-range order impossible, but also accounts for the difference between the ground-state electron density of LWG $n_{e}(\mu, \lambda)$ and that of GWC $n_{e}(\mu, 0)$. According to Eq. (16) we have $n_{e}(\mu, \lambda)-$ $-n_{e}(\mu, 0)= \pm n_{ \pm}^{g} / Q$. As is seen from Eq. (19), dimers of one sign are fully replaced by those of the opposite one as $\mu$ passes through the point $\bar{\mu}$, both $n_{+}^{g}(\bar{\mu}+0)$ and $n_{-}^{g}(\bar{\mu}-0)$ being equal to $\bar{n}_{g}=\left(u_{\xi} / u_{q}\right)^{2} \lambda^{4}$. This results in a jump of the electron density by $2 Q^{-1} \bar{n}_{g}$ at $\mu=\bar{\mu}$, i.e., in a first-order transition in $\mu$.

Another remarkable point is that $n_{ \pm}^{g}$ grows significantly as $\mu$ approaches the endpoints $\mu_{ \pm}$. Close to them a mutual repulsion of dimers (which is negligi- 
bly small for $\left|\mu-\mu_{ \pm}\right| \sim \Delta \mu$ ) should be taken into account. That problem is beyond the scope of this paper.

From the above expressions for $u_{\xi}$ and $u_{Q}$ it follows that the factor $u_{\xi}^{2} / u_{Q}^{2}$ appearing in Eq. (19) is $\sim 1$ both for $Q \sim 1$ and $Q \gg>1$, i.e., $n_{ \pm}^{g} \sim \lambda^{4}$ for $|1 \pm z| \sim 1$. According to Eq. (19) the $\mathrm{LWG}$ correlation radius $L_{g} \sim 1 / n_{+}^{g} \propto 1 / \lambda^{4}$ remains $>1$ even if $\lambda$ is not too small. This suggests that the description of LWG in terms of dimers works up to $\lambda=1 / 2$.

\section{Low-lying elementary excitations and the low-temperature thermodynamics of LWG}

\subsection{The low-lying excitation spectrum}

The low-lying LWG excitations are those whose energies are much less than the typical increase $\left(u_{c}\right)$ in the energy of the system as an interior electron of a regular claster is shifted by one site or more. They are produced by ground-state dimer displacements, and hence, they are of a many-electron nature. To find the low-lying excitation spectrum let us consider the state $|s\rangle$ created by displacement of the \pm dimer adjacent, for definiteness, to the left end of the $\alpha$-th cluster from its ground-state position, say, to the right by $s=1,2, \ldots$ steps. This occurs as $s$ electrons $m_{1 g}^{\alpha}, \ldots, m_{1 g}^{\alpha}+s-1$ are shifted to the left (-dimer) or to the right (+dimer) neighboring sites all together. The Coulomb energy of this electron «trace» is the sum

where

$$
u_{\alpha}^{ \pm}(s)=\varepsilon_{\alpha}^{ \pm}(s)+\tilde{\varepsilon}_{\alpha}^{ \pm}(s),
$$

$$
\varepsilon_{\alpha}^{ \pm}(s)=\Delta_{\bar{v}_{\alpha} \pm 1, \bar{v}_{\alpha}}\left(\bar{m}_{1 g}^{\alpha}, \bar{m}_{1 g}^{\alpha}+s\right),
$$

$\Delta_{v, v^{\prime}}$ is defined by Eq. (11);

$$
\tilde{\varepsilon}_{\alpha}^{ \pm}(s)=\tilde{\epsilon}_{ \pm}\left(\bar{m}_{1 g}^{\alpha}+s, v_{\alpha}\right)-\tilde{\epsilon}_{ \pm}\left(\bar{m}_{1 g}^{\alpha}, v_{\alpha}\right),
$$

$\tilde{\epsilon}_{ \pm}(m, v)$ is the quantity appearing in Eq. $(10) ; u(0)=$ $=0$. From this point on, we does not show a dependence on $\alpha$ or the dimer sign, provided it does not cause misunderstanding. The explicit form of $\tilde{\varepsilon}(s)$ is of no importance for further consideration; what matters is only the fact that $\tilde{\varepsilon}(s)$ takes random values within some interval $\left[-\Delta_{\lambda} / 2, \Delta_{\lambda} / 2\right]$ whose width $\Delta_{\lambda} \sim \lambda u_{c}$. The quantity $\varepsilon(s)$ can be considered as the «coordinate» of a «particle» that executes a random walk with the typical step $\delta \varepsilon \sim \lambda^{2} u_{c}<<\Delta_{\lambda}$ as the «time» $s$ increases. With an increase in $s$ energy $\varepsilon(s)$ grows as $\lambda^{2} u_{c} \sqrt{s}$, so that it becomes more than $\Delta_{\lambda}$ for $s \sim s_{\lambda} \equiv \lambda^{-2} \quad\left(s_{\lambda}<<L_{g}\right)$. Since $\varepsilon(s)$ randomly undergoes «returns» into the interval $\left[-\Delta_{\lambda} / 2, \Delta_{\lambda} / 2\right]$, there are inevitably such $s$ at which $u(s) \lesssim t$. Therefore, the low-lying excitations should be considered with regard to the electron hopping, despite the inequality $t<<u_{c}$.

At $t \neq 0$ the dimer, owing to the smallness of $t / u_{c}$, can be considered as a quasiparticle whose Hamiltonian $\mathscr{H}_{\text {dim }}$ acts in the subspace of states $|s\rangle$ (s-space). As follows from the general expression (2), it is of the form

$$
\mathcal{H}_{\operatorname{dim}}=t \sum_{s}(|s-1\rangle\langle s|+| s+1\rangle\langle s|)+\sum_{s} u(s)|s\rangle\langle s|,
$$

where the first term is the kinetic-energy operator, $u(s)$ plays the role of an effective potential; the summation is over the whole $s$-space. The state vectors of the low-lying excitations and their energies are eigenvectors $|\Psi\rangle$ of $\mathcal{H}_{\mathrm{dim}}$ and its eigenvalues $\varepsilon_{\text {exc }}$, respectively. Due to the randomness of the «potential» $u(s)$ the dimer is Anderson localized in the $s$-space, so that the eigenvectors

$$
|\Psi\rangle \equiv\left|\Psi_{l}\right\rangle=\sum_{s} \Psi_{l}(s)|s\rangle
$$

can be classified in the coordinates $l=0, \pm 1, \ldots$ of points (localization centers) about which the stationary wave functions $\psi_{l}(s)$ are centered. Since $|u(s+1)-u(s)| \sim \Delta_{\lambda}$, the Anderson parameter governing the $\left|\Psi_{l}\right\rangle$ structure is $\gamma=\Delta_{\lambda} / t \sim \lambda u_{c} / t$. It can be both less than and more than 1 despite the $t / u_{c}$ smallness. If $\gamma>>1$, the kinetic energy operator is a weak perturbartion. In such a case

$$
\psi_{l}(s)=\delta_{s l}+\mathcal{O}(1 / \gamma), \varepsilon_{\text {exc }}(l)=u(l)+\mathcal{O}(1 / \gamma),
$$

and the localization radius $r$ is thus equal to 1 . For $\gamma<<1$ the 1D Anderson localization under a weak random potential takes place. In this limit

$$
r \sim \gamma^{-2} \sim\left(t / u_{c}\right)^{2} \lambda^{-2} .
$$

For the following consideration of the thermodynamics it is necessary to know the typical separation $\varepsilon_{\text {min }}$ between the least of $\varepsilon_{\text {exc }}$ and the ground-state energy.

If $\gamma>>1$, it is determined, as is seen from Eq. (22)-(24), by the number of «returns» $R$ of the random sum $\varepsilon(s)$ to the above-mentioned domain $|\varepsilon(s)| \lesssim \Delta_{\lambda}$. Since $\varepsilon(s)$ values fall into this domain randomly, we have $\varepsilon_{\min } \sim \Delta_{\lambda} / R$. By definition

$$
R=\int_{-\Delta_{\lambda} / 2}^{\Delta_{\lambda} / 2} d \varepsilon \sum_{s=0}^{L_{g}} \mathcal{P}(s, \varepsilon),
$$

where $\mathcal{P}(s, \varepsilon) d \varepsilon$ is the probability that $\varepsilon(s)$ will fall into the interval $[\varepsilon, \varepsilon+d \varepsilon]$ after $s$ steps; for $s \gg 1$ it satisfies the diffusion equation

$$
\partial \mathcal{P} / \partial s=\mathcal{D} \partial^{2} \mathcal{P} / \partial \varepsilon^{2}
$$


with the diffusion coefficient $\mathcal{D}=\lambda^{2} D \sim(\delta \varepsilon)^{2}$ ( $D$ is given by expression (21)). Solving Eq. (27) subject to the boundary condition $P\left(s,-\Delta_{\lambda} / 2\right)=0$ (this is necessary since $\varepsilon_{\text {exc }}(l) \approx u(l)$ is positive) and substituting the solution into the expression for $R$, we find

$$
\varepsilon_{\min } \sim \lambda^{3} u_{c}<<\delta \varepsilon
$$

It should be added that the main contribution to $R$ is given by $s \sim s_{\lambda}$. Therefore, the localization center of the eigenvector with the minimal excitation energy $\varepsilon_{\text {min }}$ is generally $\sim s_{\lambda}$ distant from the ground-state position of the dimer.

For $\gamma<<1$ the localization radius according to Eq. (26) is $<<s_{\lambda}$, the eigenenergies $\varepsilon_{\text {exc }}(l)$ randomly filling a band of width $\sim t$ as $l$ varies within a domain of a length $\sim s_{\lambda}$. Therefore, in this limit

$$
\varepsilon_{\min } \sim t / s_{\lambda} \sim \lambda^{2} t .
$$

\subsection{The $L W G$ thermodynamics}

According to the above consideration, in the limiting case $T<<u_{c}$ LWG is in essence an ensemble of independent Anderson localized dimers. Their number dependence on $T$ can be ignored with accuracy to exponentially small (in $T / u_{c}$ ) corrections. The thermodynamic potential $\Omega=\Omega(\mu, T)$ of such a system is expressed in terms of a distribution of excitation energies $\varepsilon_{\mathrm{exc}}^{\alpha}(l)$ over the dimer ensemble:

$$
\begin{aligned}
\Omega=\Omega_{g} & -\mathcal{N}^{g} T \int^{\prime} \ln \left(1+\sum_{k=1} \exp \left(-\frac{\varepsilon_{k}}{T}\right)\right) \times \\
& \times W\left(\varepsilon_{1}, \varepsilon_{2}, \ldots\right) d \varepsilon_{1} d \varepsilon_{2} \ldots,
\end{aligned}
$$

where $\Omega_{g}$ is the ground-state value of $\Omega$; $W\left(\varepsilon_{1}, \varepsilon_{2}, \ldots\right)$ is the probability density that the least excitation energy of the system is $\varepsilon_{1}$, the next one is $\varepsilon_{2}$, etc.; the symbol prime means that the integration is performed over the region $\varepsilon_{1}<\varepsilon_{2}<\ldots$ from $\varepsilon_{k}=0$ $(k=1,2, \ldots)$ to the upper bound of the dimer energy spectrum (it is $\sim u_{c}$ ). The upper bound of the summation over $k \sim L_{g}$. We do not show it as it is immaterial to an accuracy of exponentially small term $\propto \exp \left(-u_{c} / T\right)$.

As was proved by Molchanov [16], there is no mutual repulsion of neighboring energy levels in 1D Anderson localized systems, unlike Wigner-Dyson statistical ensembles [17]. This holds for the energy spectrum considered, since $L_{g} \gg r$. In such a case the eigenenergies are arranged quite randomly, similar to particle coordinates in an 1D ideal gas, and $W$ is completely determined by the density of states (per dimer), $g(\varepsilon)$, with a given energy $\varepsilon$ :

$$
W\left(\varepsilon_{1}, \varepsilon_{2}, \ldots\right)=\prod_{k=1} w\left(\varepsilon_{k}, \varepsilon_{k+1}\right),
$$

where the two-level correlation function $w\left(\varepsilon, \varepsilon^{\prime}\right)$ obeys the Poisson law

$$
w\left(\varepsilon^{\prime}, \varepsilon\right)=g(\varepsilon) \exp \left(-\int_{\varepsilon^{\prime}}^{\varepsilon} g\left(\varepsilon^{\prime \prime}\right) d \varepsilon^{\prime \prime}\right) .
$$

The states density $g(\varepsilon)$ increases monotonically with a growth in $\varepsilon$ from

$$
g(0) \sim 1 / \varepsilon_{\min } \sim 1 / \lambda^{2}\left(\lambda u_{c}+t\right)
$$

to values $\sim 1 / \lambda^{4} u_{c}$. The most important dimensionless parameter governing $\Omega$ temperature dependence is thus $T g(0)$. In the limit $T g(0) \ll<1$ the thermodynamical potential can be expanded in powers of $T g(0)$. To find the $n$-th term of this series, all exponents $\exp \left(-\varepsilon_{k} / T\right)$ with $k>n$ appearing in expression (30) should be discarded. So doing, we find that to the first nonvanishing approximation $\Omega$ is of the form

$$
\Omega=\Omega_{g}-\frac{\pi^{2}}{12} \mathfrak{N}^{g} g(0) T^{2} .
$$

Consequently, both the entropy $S$ and the heat capacity $C$ per site tend to zero as $T$ with $T \rightarrow 0$. In view of Eqs. (19), (33), and (34) we obtain

$$
S, C=\frac{\pi^{2}}{6} n^{g} g(0) T \sim \lambda^{2}\left(\lambda u_{c}+t\right)^{-1} T,
$$

i.e., the proportionality coefficient is linear or quadratic in $\lambda$ depending on $\gamma>1$ or $\gamma<<1$.

In the opposit limit $T g(0)>>1$, the excitation spectrum can be considered as a continuous one. This gives

$$
\Omega=\Omega_{g}-\mathcal{N}^{g} T \ln \left(\int_{0}^{\infty} \exp (-\varepsilon / T) g(\varepsilon) d \varepsilon\right) .
$$

We have expanded the integration to infinity as the integrand is reduced rapidly for $\varepsilon \gg T$. Expression (35) shows that in the case $T g(0) \gg>1 C$ depends slightly on $T$, being $\sim n^{g}$.

\section{Super-slow relaxation in LWG}

Below we consider low-temperature $\left(T<<u_{c}\right)$ relaxation of strongly nonequilibrium LWG states formed by excitations with energies $\sim u_{c}$. The simplest excitation of this type arises if an interior electron of some regular ground-state cluster is shifted by one site from its ground-state position. Such an excitation can be considered as a pair of bound «odd» (as compared to the ground state) +dimer and -dimer. The electron 
shifted reverts to its ground-state site emitting phonons with energies $\sim u_{c}$. Obviously, this takes a microscopic time of the order of reciprocal Debye frequency. The situation is drastically changed if some external perturbation, for example, light excitation disrupts the pair, separating the «odd» \pm dimers by a sufficiently large distance (within the limits of the cluster). A dimer cannot disappear by itself, since this would cause, by virtue of dimer's topological nature, a simultaneous shift of a macroscopic number of electrons. Therefore, the «odd» dimers can only disappear by their mutual annihilation. We will show below that under slight-disorder conditions this process takes an anomalously long time*.

\subsection{The Hamiltonian and eigenvectors of a separate pair of «odd» dimers}

To the main approximation in $t / u_{c}$ the state space of the pair is a set of state vectors

$$
|\mathbf{s}\rangle=\hat{D}_{-}\left(s_{-}\right) \hat{D}_{+}\left(s_{+}+1\right)\left|\Phi_{g}\right\rangle,
$$

where $\left|\Phi_{g}\right\rangle$ is the ground-state eigenvector, $s_{-}, s_{+}$ are arbitrary integers meeting the condition $\bar{m}_{1 q}^{\alpha} \leq$ $\leq s_{-}, s_{+} \leq \bar{m}_{2 g}^{\alpha}$ (here the reduced coordinates of the regular-clauster end electrons defined in Sec. 3 are used; we denote them $\bar{m}_{1,2}$ from this point on); $\hat{D}_{-}(s)$ and $\hat{D}_{+}(s)=\hat{D}_{-}^{-1}(s)$ are the operators that shift all electrons with numbers $\geq s$ one site leftwards and rightwards, respectively. The integers $s_{ \pm}$play the role of the coordinates of the «odd» \pm dimer. The Hamiltonian of the pair $\mathcal{H}_{\text {pair }}$ can be written as

$$
\mathcal{H}_{\text {pair }}=t \sum_{\left.<\mathbf{s}^{\prime} \mathbf{s}\right\rangle}\left|\mathbf{s}^{\prime}\right\rangle\left\langle\mathbf{s}\left|+\sum_{\mathbf{s}} u(\mathbf{s})\right| \mathbf{s}\right\rangle\langle\mathbf{s}|+\Delta \mu / Q ;
$$

the first term is the operator of the kinetic energy of the pair; the symbol $\left\langle\mathbf{s}^{\prime} \mathbf{s}\right\rangle$ means summation over all nearest neighbors of $\mathbf{s}$; the «potential» energy of the pair

$$
\begin{gathered}
u(\mathbf{s})=u_{-}^{\sigma}\left(s_{-}\right)+u_{+}^{\sigma}\left(s_{+}\right), \quad \sigma=\operatorname{sgn}\left(s_{+}-s_{-}\right), \\
u_{ \pm}^{\sigma}\left(s_{ \pm}\right)= \pm \sigma \Delta_{\bar{v}, \bar{v}-\sigma}\left(\bar{m}_{1}, s_{ \pm}\right)+\tilde{\epsilon}_{ \pm}\left(s_{ \pm}\right),
\end{gathered}
$$

where $\Delta_{v, v^{\prime}}$ is the sum of random quantities defined by Eq. (11); $\tilde{\epsilon}_{ \pm}(s)=\tilde{\epsilon}_{ \pm}(s, \bar{v})$ (see Eq. (10)) oscillates randomly with the amplitude $\lambda u_{c}$. The third term in Eq. (36) is the energy of pair formation at $\lambda=0$.

The structure of Hamiltonian (36) is akin to that of the one-dimer Hamiltonian (25). It immediately follows that the +dimer and -dimer of a pair are both An- derson localized in the s-representation, the localization radius $r$ being $\sim 1+\left(t / \lambda u_{c}\right)^{2}$. Classifying the pair's eigenvectors $|\Phi\rangle$ by coordinates $l_{-}, l_{+}$of the \pm dimer localization centers, we can write $|\Phi\rangle$ as

$$
|\Phi\rangle=\left|\Phi_{1}\right\rangle=\sum_{\mathbf{s}} \psi_{1}(\mathbf{s})|\mathbf{s}\rangle
$$

Here $\mathbf{l}=\left\{l_{-}, l_{+}\right\}$, the summation is over all $\mathbf{s}, \psi_{\mathbf{l}}(\mathbf{s})$ is the pair's wave function, which is localized in the $\mathbf{s}$-space region $|\mathbf{l}-\mathbf{s}| \lesssim r$. In the general case that the pair's length $l_{p}=\left|l_{-}-l_{+}\right| \gg r$ one can replace $\sigma$ in Eq. (37) with sgn $\left(l_{+}-l_{-}\right)$. This allows $\psi_{1}(\mathbf{s})$ to be factorized:

$$
\psi_{1}(\mathbf{s})=\varphi_{l_{-}}\left(s_{-}\right) \varphi_{l_{+}}\left(s_{+}\right),
$$

the \pm dimer wave function $\varphi_{l_{ \pm}}\left(s_{ \pm}\right)$satisfying the Schrödinger equation

$$
\begin{gathered}
t\left(\varphi_{l_{ \pm}}\left(s_{ \pm}+1\right)+\varphi_{l_{ \pm}}\left(s_{ \pm}-1\right)\right)+u_{ \pm}^{\sigma}\left(s_{ \pm}\right) \varphi_{l_{ \pm}}\left(s_{ \pm}\right)= \\
=E_{ \pm}\left(l_{ \pm}\right) \varphi_{l_{ \pm}}\left(s_{ \pm}\right) .
\end{gathered}
$$

The eigenenergy $E_{p}(1)$ of the Hamiltonian (36) is related to the eigenvalues $E_{ \pm}\left(l_{ \pm}\right)$by the formula

$$
E_{p}(\mathbf{l})=E_{-}\left(l_{-}\right)+E_{+}\left(l_{+}\right)+\Delta \mu / Q>0 .
$$

If $\gamma \gg>1 \quad(r=1)$, we have $E_{-}\left(l_{-}\right)+E_{+}\left(l_{+}\right) \approx$ $\approx \Delta_{\bar{v}, \bar{v}_{-1}}\left(l_{-}, l_{+}\right)$or $\Delta_{\bar{v}, \bar{v}_{+1}}\left(l_{+}, l_{-}\right)$depending on whether $l_{-}<l_{+}$or $l_{-}>l_{+}$. For $\gamma \lesssim 1\left(r=\left(t / \lambda u_{c}\right)^{2}\right)$ the $\operatorname{sum} E_{-}\left(l_{-}\right)+E_{+}\left(l_{+}\right)$represents $\Delta_{\bar{v}, \bar{v} \mp 1}\left(l_{\bar{\mp}}, l_{ \pm}\right)$behavior in outline. Therefore, $E_{p}(\mathbf{l})$ as a function of $l_{p}$ undergoes, together with $\Delta_{v, v \pm 1}$, random alternating-sign fluctuations whose amplitude grows as $\lambda^{2} u_{c} l_{p}^{1 / 2}$ with increasing $l_{p}$. The minimal energy $\bar{E}_{p}$ of the energy spectrum of the pair is generally $\sim u_{c}$, the pair's length in the state $\left|\Phi_{1}\right\rangle$ with $E_{p}(1)=\bar{E}_{p}$ being $\sim L_{g}$.

\subsection{Transition frequencies}

At $T=0$ the annihilation of the pair is by phonon emission. First one should consider the frequency $v(\mathbf{1})$ of the direct quantum transition $\left|\Phi_{1}\right\rangle \rightarrow\left|\Phi_{g}\right\rangle$ for an arbitrary $\left|\Phi_{1}\right\rangle$. According to Fermi's golden rule, we have

$$
v(1) \propto \overline{|A(1)|^{2}},
$$

where

$$
A(1)=\left\langle\Phi_{1}\left|H_{e-\mathrm{ph}}\right| \Phi_{g}\right\rangle
$$

is the amplitude of the transition $\left|\Phi_{1}\right\rangle \rightarrow\left|\Phi_{g}\right\rangle, H_{e-\mathrm{ph}}$ is the Hamiltonian of the electron-phonon interac-

* A pair of separated «odd» dimers can also be thought of as an excitation arising as some subcluster of a regular cluster is shifted as a whole by one site. As will be clear from the following, the lifetime of an excitation produced by a shift of the subcluster by more than one site exceeds the lifetime of a pair of dimers. 
tion, and the line over $|A|^{2}$ symbolizes averaging over all finite phonon states with energy $E_{p}(1)$.

Since the electron-phonon interaction is of a local character, only $\psi_{1}(\mathrm{~s})$ with $\left|s_{-}-s_{+}\right| \sim 1$ or $s_{-}=s_{+}$contribute to expression (43). To estimate $v(\mathbf{1})$ it is sufficient to consider $\psi_{1}(s, s)$, using the factorized expression (39). Since the typical $l_{p}$ is $L_{g}$, it is necessary to clarify the asymptotic behavior of $\varphi_{l_{ \pm}}(s)$ for $\left|l_{ \pm}-s_{ \pm}\right| \sim L_{g}$. So doing, one should take into consideration that the difference $u_{ \pm}^{\sigma}\left(s_{ \pm}\right)-u_{ \pm}^{\sigma}\left(l_{ \pm}\right)$, being a random sum of $\left|l_{+}-s_{+}\right|$terms, undergoes fluctuations with the typical amplitude $\Delta u \sim \lambda^{2} u_{c}\left|l_{ \pm}-s_{ \pm}\right|^{1 / 2}$. It is these effective-potential fluctuations rather than the dimer Anderson localization that govern the decreasing of $\varphi_{l_{ \pm}}(s)$ when $\Delta u$ exceeds $\Delta_{\lambda}^{*} \sim \lambda u_{c}+t$. The latter is the typical spread of eigenenergies of a reduced Schrödinger equation which would come from Eq. (40) with the randomly oscillating bounded function $\tilde{\epsilon}_{ \pm}\left(s_{ \pm}\right)$(see Eqs. (10) and (37)) in place of $u_{ \pm}^{\sigma}\left(s_{ \pm}\right)$. This takes place for $\left|l_{ \pm}-s_{ \pm}\right| \gtrsim s_{\lambda}^{*}=\left(\Delta_{\lambda}^{*} / \lambda^{2} u_{c}\right)^{2}$ $\left(s_{\lambda}^{*}<<L_{g}\right)$. In such a case $\varphi_{l_{+}}(s)$ is nonzero owing to dimer tunneling through the fluctuation barrier. Basing on the Schrödinger equation (40), we find that in the above forbidden region

$$
\varphi_{l_{ \pm}}(s)=B\left(\frac{t}{\left|l_{ \pm}-s_{ \pm}\right|^{1 / 2}}\right)^{b\left|l_{ \pm}-s_{ \pm}\right|},
$$

where the factor

$$
B=\exp \left(-b_{1} \frac{s_{\lambda}^{*}}{r} \ln \frac{\Delta_{\lambda}^{*}}{t}\right),
$$

results from falling of the dimer wave function (over a distance $\sim s_{\lambda}^{*}$ ) due to the Anderson localization; $b, b_{1}$ are some constants $\sim 1$. Here we omit a pre-exponential factor $\sim 1$. Substitution of expression (40) in Eqs. (39) and (42) gives

$$
v(\mathbf{l})=v_{0} l_{p} B^{4} \exp \left(-\theta l_{p}\right), \theta=4 b \ln \frac{\lambda^{2} l_{p}^{1 / 2}}{t} .
$$

The pre-exponential factor $v_{0} \lesssim \omega_{D}$ is expressed in terms of electron-phonon interaction characteristics; $\omega_{D}$ is the Debye frequency. The expression holds for $l_{p} \gtrsim s_{\lambda}^{*}$. The exponential decline of $v(\mathbf{l})$ with increasing $l_{p}$ is in essence of a many-electron origin. It becomes clear if one recalls that in the state $\left|\Phi_{1}\right\rangle$ there are $\sim l_{p} \gg 1$ electrons shifted about their groundstate positions. The annihilation of a pair's dimers occurs if all electrons shifted revert to the ground-state sites simultaneously. Naturally, this results in small $v(1)$ values.

The foregoing argument can be easily extended to find the frequencies $v\left(\mathbf{1}, \mathbf{l}^{\prime}\right)$ of quantum transitions
$\left|\Phi_{1}\right\rangle \rightarrow\left|\Phi_{1^{\prime}}\right\rangle$, which occur with emission of phonons with energies $E_{p}(\mathbf{l})-E_{p}\left(\mathbf{l}^{\prime}\right)$. The expression for $v\left(1,1^{\prime}\right)$ is of the form

$$
v\left(\mathbf{1}, \mathbf{1}^{\prime}\right)=v_{0} d_{ \pm} B^{4} \exp \left(-\theta_{ \pm} d_{ \pm}\right), \theta_{ \pm}=4 b \ln \frac{\lambda^{2} d_{ \pm}^{1 / 2}}{t}
$$

where $d_{ \pm}=\left|l_{ \pm}-l_{ \pm}^{\prime}\right|$. It is implied that only one difference, $d_{-}$or $d_{+}$, is nonzero. Otherwise, the transition frequencies are exponentially small as compared with those given by Eq. (46).

\subsection{Relaxation of a separate pair of «odd» dimers}

To find the time $\tau_{p}$ of the annihilation of a pair, one should keep track of how a pair's density matrix $\hat{\rho}=\rho(1, \tau) \delta_{1,1^{\prime}}$ varies with time $\tau$. The quantities $\rho(1, \tau)$ that are the probabilities of finding the pair in the states $\left|\Phi_{1}\right\rangle$ at an instant of time $\tau$ satisfy a Pauli-like kinetic equation

$$
\frac{\partial \rho(1, \tau)}{\partial \tau}=\sum_{1}>v\left(\mathbf{l}, \mathbf{l}^{\prime}\right) \rho\left(\mathbf{l}^{\prime}\right)-\left(v^{<}(\mathbf{l})+v(\mathbf{l})\right) \rho(\mathbf{l}, \tau),
$$

where

$$
v^{<}(1)=\sum_{1^{\prime}}{ }^{<} v\left(1,1^{\prime}\right)
$$

Here symbols $>$ and $<$ mean that the summation extends only to $\mathbf{l}^{\prime}$ for which $E_{p}\left(\mathbf{l}^{\prime}\right)-E_{p}(\mathbf{l})>0$ and $<0$, respectively. If $l_{p}, d_{ \pm} \sim r$, the transition frequencies $v(\mathbf{l}), v\left(\mathbf{l}, \mathbf{1}^{\prime}\right) \sim v_{0}$; for $l_{p}, d_{ \pm}>r$ they are determined by Eqs. (45) and (46).

The annihilation of a pair is governed by two equations. One is for the probability of existence of the pair at an instant $\tau$,

$$
P(\tau)=\sum_{1} \rho(1, \tau)
$$

(the summation is over all 1). Another is for the energy-average value

$$
\eta(\tau)=\sum_{1} \tilde{E}_{p}(1) \rho(1, \tau)
$$

where $\tilde{E}_{p}(\mathbf{1})$ is the eigenenergy measured from the minimal energy $\bar{E}_{p}$. Both equations follow immediately from Eq. (47):

$$
\begin{gathered}
d P / d \tau=-\sum_{1} v(\mathbf{l}) \rho(1, \tau) \\
\frac{d \eta(\tau)}{d \tau}=-\sum_{1,1^{\prime}}<\left(E_{p}(\mathbf{1})-E_{p}\left(\mathbf{l}^{\prime}\right)\right) v\left(1, \mathbf{l}^{\prime}\right) \rho(1, \tau)- \\
-\sum_{1} v(1) \tilde{E}_{p}(1) \rho(1, \tau)<0
\end{gathered}
$$


These equations show that the zero-temperature lifetime of the pair $\tau_{p}$ is conditioned by which of two processes is the faster: a fall in $\eta(\tau)$ caused by $\left|\Phi_{1}\right\rangle \rightarrow\left|\Phi_{1^{\prime}}\right\rangle$ transitions, or decreasing of $\eta(\tau)$ together with $P(\tau)$ as a result of direct transitions $\left|\Phi_{1}\right\rangle \rightarrow\left|\Phi_{g}\right\rangle$. In the first case the annihilation occurs in two stages: in the first stage $P(\tau)$ remains close to unity, while $\eta(\tau)$ approaches a vicinity of zero; in the next stage $P(\tau)$ vanishes, $\rho(1, \tau)$ being nonzero only at such 1 for which $\tilde{E}_{p}(\mathbf{l}) \approx 0$. For this scenario $\tau_{p}$ does not depend on the initial state $\rho(1,0)=\delta_{1_{0}} 1$. In the second case a pronounced dependence of $\tau_{p}$ on the initial $\mathbf{l}=\mathbf{l}_{0}$ should be expected

In Appendix it is shown that

$$
\tau_{p}=1 / \min _{1}\left(v^{<}(\mathbf{1})+v(1)\right)
$$

(the minimum in $\mathbf{1}$ is implied), i.e., $\tau_{p}$ does not depend on $\mathbf{l}_{0}$, and hence, the first of the above-mentioned scenarios takes place. In such a case $\tilde{E}_{p}(1)$ value at the minimum point $\mathbf{l}=\mathbf{1}$ min of the function $v^{<}(1)+v(1)$ is bound to be among a few eigenenergies closest to zero. This can be justified on the following grounds. As follows from item 5.1, $\tilde{E}_{p}(1)$ as a function of $\mathbf{l}$ executes a random walk with a diffusion coefficient of the order of $\mathcal{D}$ appearing in Eq. (27). Simple estimates based on this fact show that the number $N_{1}$ of eigenenergies between a given $\tilde{E}_{p}(\mathbf{1})$ and zero is $\sim \tilde{E}_{p}(1) \sqrt{L_{g} / \mathcal{D}} \sim \tilde{E}_{p}(1) / \lambda^{4} u_{c}$. Since, on the one hand, the mean separation of points $\mathbf{l}^{\prime}$ at which $E_{p}\left(\mathbf{l}^{\prime}\right)<E_{p}(\mathbf{l})$ is $\sim L_{g} / N_{1}$, and on the other hand, $v\left(1, \mathbf{l}^{\prime}\right)$ falls exponentially as $d_{+}$increases, we see (in view of the definition (48)) that the less is $N_{1}$ the less is $v^{<}(\mathbf{l})+v(\mathbf{l})$, i.e., at the minimum point $\mathbf{l}$ min we really have $N_{1}$ min $\sim 1$. Hence $\left|l_{+}{ }^{\min }-l_{-}{ }^{\min }\right| \sim L_{g}$. Taking into account this fact together with Eqs. (45) and (46), we arrive thus to conclusion that

$$
\left|\ln v_{0} \tau_{p}\right| \sim \ln \left(u_{c} / t\right) \lambda^{-4} .
$$

This $\tau_{p}$ value is anomalously great: it is more than $\omega_{D}^{-1}$ by tens of orders of magnitude even if $\lambda$ is not too small $(\lambda=1 / 3-1 / 4)$.

At finite temperatures activation transitions caused by phonon absorbtion can provide annihilation of dimers by bringing them at distances $\sim r$, whatever the initial $l_{p}$ may be. If the pair's length $l_{p}>s_{\lambda}^{*}$, drawing dimers into the proximity requires them to overcome a fluctuation energy barrier $\sim \lambda^{2} u_{c} l_{p}{ }^{1 / 2}$. Within an exponential factor the activation transition frequency is

$$
v_{\text {act }}=-\exp \left(-\lambda^{2} u_{c} l_{p}{ }^{1 / 2} / T\right)
$$

In the temperature region $T<<\Delta_{\lambda}^{*}$ the pre-exponential factor omitted is determined by the Mott's variable-range-hopping [18] over distances $\lesssim s_{\lambda}^{*}$. For the typical $l_{p} \sim L_{g}$ we obtain for the activation time of the dimer annihilation $\tau_{\text {act }}=1 / v_{\text {act }}$

$$
\tau_{\text {act }} \propto \exp \left(u_{c} / T\right)
$$

The time $\tau_{\text {act }}$ becomes less than the tunnel relaxation time Eq. (52) when $\lambda<\left(T / u_{c}\right)^{1 / 4}$. At helium temperatures and in the most realistic case $u_{c} \sim 10^{2}-$ $10^{3} \mathrm{~K}$ this gives $\lambda<1 / 5$. However, $\tau_{\text {act }}$ remains anomalously great.

\subsection{Relaxation of an ensemble of odd-dimer pairs}

Experimentally, it is possible to create (e.g., by optical excitation) a set of \pm dimer pairs whose density is $>n_{g}^{ \pm}$, but is $<<n_{e}$. It should be elucidated how the density of pairs $n(\tau)$ or, quite the same, the concentration of alternating «odd» + and -dimers reduces with time $\tau$. The decrease in $n(\tau)$ is expected to be so slow (this is verified below) that the ensemble of pairs has time to come to the partial equilibrium corresponding to current $n(\tau)$. (In such a case the energy of the state at an instant $\tau$ is approximately equal to the energy Eq. (18) at $n_{ \pm}=n(\tau)$.) This allows a «reduction of the description», so that we may restrict ourselves to consideration of the differential equation

$$
d n / d \tau=-\omega(n) n,
$$

wherein the transition frequency $\omega(n)$ comes from expression (45) (the dimer tunneling prevails) or from Eq. (53) (the activation prevails) by substitution of $L(\tau)=1 / n(\tau)$ for $l_{p}$. Correspondingly, there are two different asymptotic dependences of $n$ on $\tau$. If the activation can be neglected, we have

$$
n=\frac{b \ln \ln v_{0} \tau}{\ln v_{0} \tau} .
$$

In the case that the activation is paramount, Eq. (54) gives

$$
n=\left(\lambda^{2} u_{c} / T\right)^{2} \ln ^{-2} v_{0} \tau .
$$

Expressions (55) and (56) have been written to the main logarithmic approximation. They hold for $L(\tau)>>s_{\lambda}^{*}, L(\tau) \lesssim L_{g}$. The logarithmic decreasing of $n(\tau)$ with time is a salient feature of LWG.

The super-slow relaxation in LWG and its logarithmic time-dependence recall to some extent those in spin glass [13], which features a nonergodic behavior and an infinite spectrum of relaxation times caused by a macroscopic multi-valley degeneracy of the ground state. As is known, a spin-glass valley is a state whose energy is close to the ground-state one, but its space structure differs from that of the ground state macroscopically. Transition from the valley to the ground state takes place only if a macroscopically great number of spin flips occur simultaneously. In the LWG the 
analogues of the spin-glass valleys are the above-mentioned electron subclusters containing a great (but by no means macroscopic) numbers of electrons. This gives grounds to consider the «spin-glass» LWG features as a quasi-nonergodic behavior.

The above consideration is extended to the case of an arbitrary rational $n_{e}=P / Q$ with $P \neq 1$ (see Secs. 1 and 2) almost without modifications. The only difference is that in the general case the discrete solitons (GWC defects of compression and rarefaction) arising in the system with a change of the electron number are not simple \pm dimers, but more complicated structures [10]. However, this is immaterial since only the formation energy of the GWC defect $\pm\left(\mu_{ \pm}-\mu\right) / Q$ $\left(\mu_{+}=\mu_{+}(P / Q)\right)$ is relevant.

The long-range order in GWC is also broken if the system is exposed to an external weak random potential. The above line of argument can be extended to this situation without change. Bearing this in mind, it is easy to find that the ground-state dimer concentration depends on the potential amplitude quadratically.

\section{Conclusion}

Our results show that the low-temperature thermodynamics and kinetics of LWG are fully described in terms of GWC point defects of a many-electron origin (discrete solitons). It is the soliton nature of these defects (for definiteness, we will consider them to be dimers) that imparts to the LWG its distinctive features differing qualitatively from those of the known Wigner glass: the spontaneous dimer formation in the ground state caused by an arbitrarily weak disorder of the host lattice, first-order phase transitions in $\mu$ at zero temperature, and the almost nonergodic macroscopic behavior. These facts raise questions inviting further investigation. Below we outline some of them.

First of all, the transport and high-frequency properties of LWG should be clarified, taking into account that each \pm dimer carries the fractional charge $e^{*}= \pm e / Q$ ( $e$ is the free electron charge $)$. Conduction in $\mathrm{LWG}$ is by dimer transfer over distances $\sim L_{g}=1 / n_{+}^{g}(\mu)$. This requires dimers to overcome a fluctuation barrier of the order of the energy of dimer formation $u_{ \pm}(\mu)$ (see Eq. (15)), so that the LWG static conductivity $\sigma_{\mathrm{LWG}}$ is expected to be proportional to

$$
\exp \left(-L_{g}(\mu) \ln \frac{u_{ \pm}(\mu)}{t}\right) \text { or to } \exp \left(-\frac{u_{ \pm}(\mu)}{T}\right)
$$

depending on which of the two mechanisms prevails: dimer tunneling through the barrier or the activation transitions. Since $L_{g}(\mu)$ and $u_{ \pm}$fall when $\mu$ approaches the endpoints $\mu_{ \pm}$of the devil's-staircase intervals (see Eqs. (15) and (19)), $\sigma_{\mathrm{LWG}}$ is bound to show pronounced splashes in vicinities of $\mu_{ \pm}$. These giant oscillations of $\sigma_{\mathrm{LWG}}$ as a function of $\mu$ are not only of interest by themselves, but also can be an effective experimental instrument for investigating the super-slow (logarithmic) relaxation of a strongly nonequilibrium LWG state. At a fixed $\mu$ a proper way to observe the relaxation is keeping track of photoabsorbtion of low-intensity light ${ }^{\star}$.

Since the fluctuation energy barriers responsible for LWG resistance cover long intervals of the order of $L_{g} \sim 1 / \lambda^{4}$, a noticeable deviation from Ohm's law is expected to arise even for low strengths $\mathcal{E}$ of the applied electric field. Namely, this takes place if $e^{*} \mathcal{E} L_{g}$ becomes comparable with the typical barrier height $u_{ \pm}$. The electric intensities that meet this condition, $\mathcal{E} \sim \lambda^{4} u_{ \pm}^{3} / e$, rapidly decline as $\lambda$ decreases. They are especially low in vicinities of the endpoints of the devil's-staircase intervals. It is remarkable that this nonlinear effect can be pronounced even though the LWG conductance is low.

Results obtained in Sec. 3 (see also the note at the end of Sec. 5) show that the length of space correlation in $L W G L_{g} Q$ is in inverse proportion to the square of the typical energy of a random static perturbation, i.e., it behaves in the same way as the correlation length in continuous Wigner glass [12], wherein the inverse quadratic dependence takes place for any dimension $d \leq 3$. This raises a very interesting question whether such an accordance between discrete (lattice) electron systems and continuous ones remains for $d>1$, and moreover, whether discrete electron systems with $d>1$ are unstable with respect to an arbitrarily weak random external perturbation. Our preliminary studies leads us to the conclusion that, owing to the phenomenon of the effective lowering of dimension mentioned in Sec. 1, the 2D modification of GWC is stable in this sense: at a given $\mu$ a random distortion of the host lattice breaks the long-range order of this system only if the disorder parameter exceeds some critical value, which vanishes at the endpoints of the devil's-staircase intervals. This suggests that macroscopic behavior of a 2D electron lattice system on a weakly disordered host lattice has nothing in common with that of 2D Wigner glass.

We are going to carry out detailed studies of the above problems in the immediate future.

The authors are grateful to V.D. Natsik and L.A. Pastur for fruitful discussions.

* Matrix elements of photoinduced one-step dimer transitions are not small. 


\section{Appendix}

To find the general expression for the relaxation time $\tau_{p}$ of a pair of dimers, it is convenient to enumerate eigenenergies $E(1)$ in increasing order: $E_{i}=$ $=E\left(\mathbf{1}_{i}\right)<E_{i+1}, i=1,2, \ldots$; energy $E_{1}$ is the least of $E(1)$. In these terms the kinetic equation (47) takes the form

$$
d \rho(\tau) / d \tau=\hat{v} \rho(\tau)
$$

where $\boldsymbol{\rho}=\left(\rho_{1}, \rho_{2}, \ldots\right), \rho_{i}(\tau) \equiv \rho\left(\mathbf{1}_{i}, \tau\right)$,

$$
\hat{v} \rho(\tau)=\sum_{k \geq i} v_{i k} \rho_{k}(\tau)
$$

and $v_{i k}$ is a triangular matrix:

$$
v_{i k}=\left\{\begin{array}{l}
v\left(\mathbf{1}_{i}, \mathbf{1}_{k}\right), k>i ; \quad v_{i k}=0, \quad k<i ; \\
-v\left(\mathbf{l}_{i}\right)-\sum_{i^{\prime}=1}^{i} v_{i^{\prime} i}, \quad k=i .
\end{array}\right.
$$

The general solution of Eq. (A.1) can be expressed in terms of eigenvectors $\mathbf{f}_{j}$ and $\mathbf{f}_{j}^{\prime}(j=1,2, \ldots)$ of the operator $\hat{v}$ and the conjugate one $\hat{v}^{\prime}\left(v_{i k}^{\prime}=v_{k i}\right)$, respectively. They satisfy the equations

$$
\hat{v} \mathbf{f}_{j}=\Lambda_{j} \mathbf{f}_{j}, \hat{v}^{\prime} \mathbf{f}_{j}^{\prime}=\Lambda_{j} \mathbf{f}_{j}^{\prime},
$$

where

$$
\Lambda_{j}=v_{j j}=-v\left(\mathbf{l}_{j}\right)-\sum_{i=1}^{j} v_{i j}<0
$$

are the eigenvalues of the operators $\hat{v}$ and $\hat{v}^{\prime}$.

Taking into account that $\left\langle\mathbf{f}_{j}^{\prime} \mid \mathbf{f}_{j^{\prime}}\right\rangle=\delta_{j j^{\prime}} \quad(\langle\mathbf{a} \mid \mathbf{b}\rangle$ means the scalar product $\sum_{i} a_{i} b_{i}$ here and further on), from Eqs. (A.1) and (A.4) we obtain

$$
\boldsymbol{\rho}(\tau)=\sum_{j}\left\langle\mathbf{f}_{j}^{\prime} \mid \boldsymbol{\rho}_{0}\right\rangle \exp \left(\Lambda_{j} \tau\right) \mathbf{f}_{j}
$$

where $\rho_{0}=\rho(0)$.

Since $\Lambda_{j}$ are all negative, this expression tends asymptotically to

$$
\boldsymbol{\rho}(\tau)=\left\langle\mathbf{f}_{j}^{\prime} \mid \boldsymbol{\rho}_{0}\right\rangle \exp \left(\Lambda_{j}{ }^{*} \tau\right) \mathbf{f}_{j}{ }^{*}
$$

as $\tau \rightarrow \infty$. Here $j^{*}$ is the number of the eigenvalue with the least modulus. (As follows from the reasoning after formula (51), $j^{*} \sim 1$. Hence, $\tau_{p}=1 /\left|\Lambda_{j}^{*}\right|$. This coincides with expression (51).
As to the prefactor $\left\langle\mathbf{f}_{j}^{\prime}{ }^{*} \mid \boldsymbol{\rho}_{0}\right\rangle$, it is easy to show (in view of the triangular form of the matrix $v_{i k}$ ) that it is $\sim 1$ for all $\rho_{0}$ of the form $\rho_{0 i}=\delta_{i i_{0}}$, except $i_{0}<j^{*}$. In the latter (very special) case the relaxation time is less than $1 / \mid \Lambda_{j}{ }^{*}$, but its logarithm is of the same order of magnitude.

1. B.I. Shklovskii and A.L. Efros, Electronic Properties of Doped Semiconductors, Springer, Berlin (1984).

2. M.S. Bello, E.I. Levin, B.I. Shklovskii, and A.L. Efros, Sov. Phys. JETP 80, 1596 (1981) [JETP 53, 822 (1981)].

3. J. Hubbard, Phys. Rev. B17, 494 (1978).

4. C.J. Adkins, A.I. Yakimov, A.V. Dvurechenskii, V.V. Kirienko, Yu.I. Yakovlev, and A.I. Nikiforov, Phys. Rev. B61(16), 10868 (2000).

5. $2 D$ Electron Systems on Helium and Other Substrates, E.Y. Andrei (ed.), Kluwer, New York (1997), and references there.

6. H. Nejoh and M. Aono, Appl. Phys. Lett. 64(21), 2803 (1994); R.G. Osifchin, W.J. Mahoney, J.D. Bielefeld, R.P. Andres, J.I. Henderson, and C.P. Kubiak, Superlattices and Microstructures 18(4), 283 (1995).

7. P. Bak and R. Bruinsma, Phys. Rev. Lett. 49, 249 (1982).

8. Ya.G. Sinai and S.E. Burkov, Usp. Mat. Nauk 38, 205 (1983).

9. V.V. Slavin and A.A. Slutskin, Phys. Rev. B54(11), 8095 (1996).

10. A.A. Slutskin, V.V. Slavin, and H.A. Kovtun, Phys. Rev. B61(20), 14184 (2000).

11. A.I. Larkin, Sov. Phys. JETP 31, 784 (1970).

12. Y. Imri and S.-K. Ma, Phys. Rev. Lett. 35, 1399 (1975).

13. M. Mezard, G. Parisi, and M. Verasoro, Spin Glass Theory and Beyond, World Scientific, Singapore-New Jersey - Hong Kong (1987).

14. S.L. Ginzburg, Irreversible Phenomema of Spin Glasses, Nauka, Main Editorial Board for Phys. and Math. Literature, Moscow (1989).

15. W. Feller, An Introduction to Probability Theory and its Applications, New York John Wiley and Sons - London Chapman and Hall, Volume I (1958).

16. S. Molchanov, Comm. Math. Phys. 78, 429 (1981).

17. K. Efetov, Supersymmetry in Disorder and Chaos, Cambridge University Press (1999).

18. N.F. Mott and E.A. Davis, Electron Processes in Non-crystalline Materials, Clarendon Press, Oxford (1979).

19. M. Pepper, J. Phys. C12, L617 (1979). 